\title{
Rim and Flame Signs: Postgadolinium MRI Findings Specific for Non-CNS Intramedullary Spinal Cord Metastases
}

\author{
J.B. Rykken, F.E. Diehn, C.H. Hunt, L.J. Eckel, K.M. Schwartz, T.J. Kaufmann, J.T. Wald, C. Giannini, and C.P. Wood
}

\begin{abstract}
BACKGROUND AND PURPOSE: No highly specific MR imaging features distinguishing ISCMs from primary cord masses have been described. Our purpose was to retrospectively compare peripheral enhancement features on postgadolinium MR imaging of ISCMs with primary intramedullary cord masses.

MATERIALS AND METHODS: A consecutive group of patients with firmly diagnosed ISCM (45 patients with 64 ISCMs) and a comparison group with consecutive pathologically proved primary intramedullary spinal cord masses (64 patients with 64 primary spinal cord masses: ependymoma, astrocytoma, hemangioblastoma, ganglioglioma, and cavernous malformation) were included. MR images were evaluated for 2 specific signs on postgadolinium images: a "rim" sign (more intense thin rim of peripheral enhancement around an enhancing lesion) and "flame" sign (ill-defined flame-shaped region of enhancement at the superior/inferior lesion margins). The frequency of rim and/or flame signs in ISCMs and primary cord masses was compared ( $\chi^{2}$ test). For ISCMs, the maximal dimension of the enhancing lesion was correlated with the presence of rim or flame signs ( $t$ test).

RESULTS: Rim and flame signs, alone and in combination, were seen more frequently in ISCMs than in primary cord masses $(P<.0001$ for each). Specificity and sensitivity, respectively, for diagnosing ISCMs among spinal cord masses on a per-patient basis were the following: rim sign, 97\%, 47\%; flame sign, 97\%, 40\%; at least 1 sign, 94\%, 60\%; and both signs concurrently, $100 \%, 27 \%$. In the ISCM group, the presence of either a rim or flame sign correlated with a larger measured maximum enhancing lesion size $(P=.0065$ and $P=.0012$, respectively).
\end{abstract}

CONCLUSIONS: The rim and flame signs are common in and specific for ISCM and are rare in primary spinal cord masses.

ABBREVIATIONS: $\mathrm{ABR}=$ American Board of Radiology; $I \mathrm{SCM}=$ intramedullary spinal cord metastasis; $\mathrm{WHO}=$ World Health Organization

SCMs are rare. Postmortem series report a prevalence of $0.9 \%-$

$2.1 \%$ in patients with cancer. ${ }^{1,2}$ However, ISCMs are being observed more commonly than in the past, with contributing factors including the increasing use and availability of MR imaging and more effective cancer therapies. ${ }^{3-5} \mathrm{MR}$ imaging is the diagnostic imaging technique of choice for ISCMs, yet large case series with detailed descriptions of MR imaging features of these lesions are lacking.

To our knowledge, no highly specific MR imaging features of ISCMs that could distinguish them from primary cord masses have been described. Even in the MR imaging era, these lesions are

Received June 3, 2012; accepted after revision July 9.

From the Department of Radiology, Division of Neuroradiology (J.B.R., F.E.D.

C.H.H., L.J.E., K.M.S., T.J.K., J.T.W., C.P.W.), and Department of Pathology (C.G.), Mayo Clinic, Rochester, Minnesota.

Paper previously presented at: Annual Meeting of the American Society of Spine Radiology, February 16-19, 2012; Miami Beach, Florida.

Please address correspondence to Felix E. Diehn, MD, Department of Radiology, Mayo Clinic, 200 1st St SW, Rochester, MN 55905; e-mail: Diehn.felix@mayo.edu

http://dx.doi.org/10.3174/ajnr.A3292 occasionally biopsied for diagnostic purposes. We have anecdotally observed 2 potentially distinguishing postgadolinium MR imaging features at the peripheral aspects of ISCMs. The purpose of the current study was to retrospectively compare the peripheral enhancement features on postgadolinium MR imaging of ISCMs and primary intramedullary cord masses.

\section{MATERIALS AND METHODS}

Institutional review board approval with waived consent was obtained for this Health Insurance Portability and Accountability Act-compliant retrospective research study.

\section{Subjects}

The radiologic, clinical, surgical, and pathologic data bases at our single institution were searched for a group of consecutive patients with ISCMs ( $n=58,1999$ to 2011). Patients with lack of available gadolinium-enhanced pretreatment digital MR imaging examinations, and patients in whom alternative diagnoses were being considered clinically and in whom ISCM was never formally diagnosed were excluded. For any patient with pathologi- 

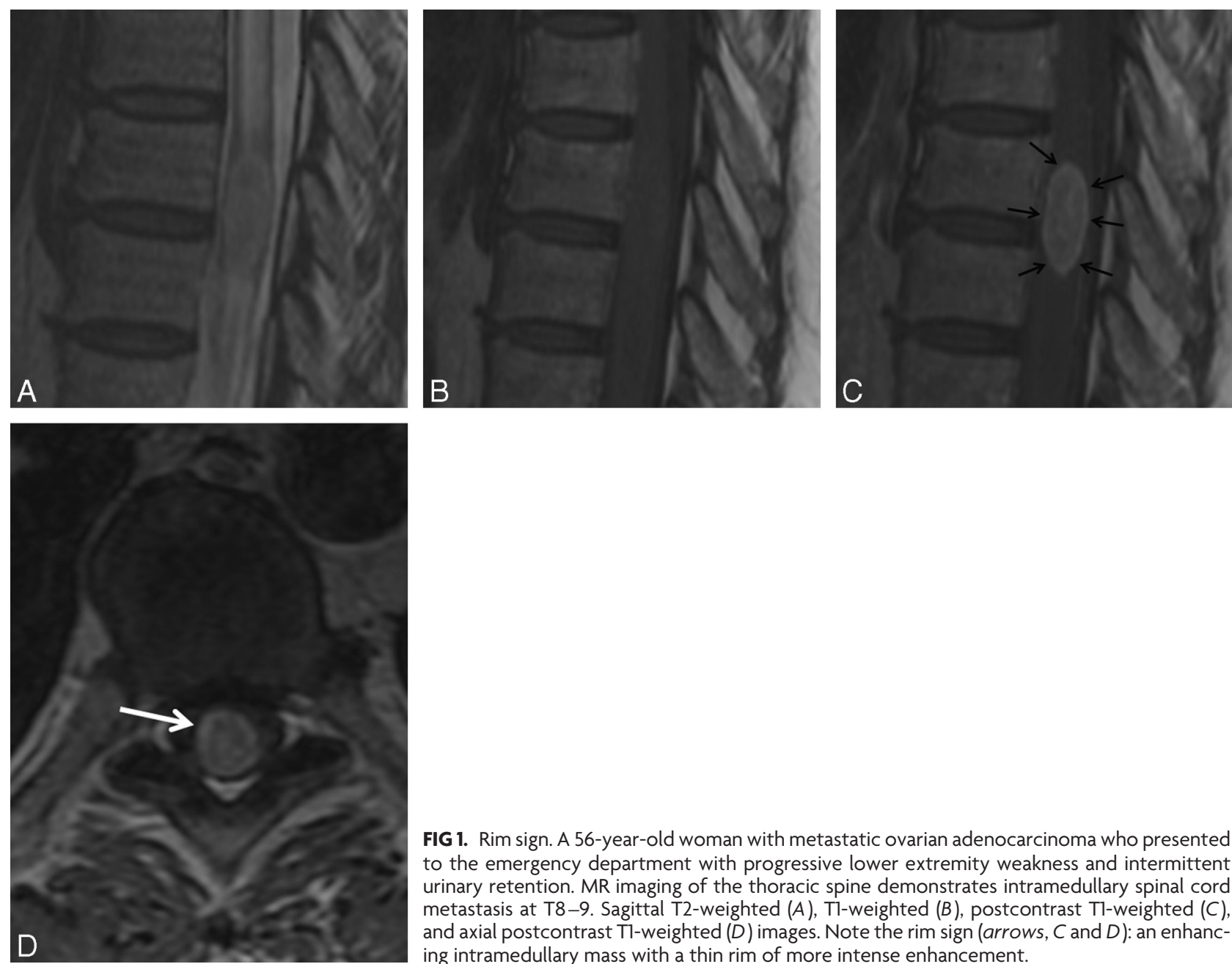

FIG 1. Rim sign. A 56-year-old woman with metastatic ovarian adenocarcinoma who presented to the emergency department with progressive lower extremity weakness and intermittent urinary retention. MR imaging of the thoracic spine demonstrates intramedullary spinal cord metastasis at T8-9. Sagittal T2-weighted (A), T1-weighted (B), postcontrast T1-weighted (C), and axial postcontrast T1-weighted $(D)$ images. Note the rim sign (arrows, $C$ and $D$ ): an enhancing intramedullary mass with a thin rim of more intense enhancement.

cally proved ISCM, the primary reason (diagnostic or therapeutic) for cord mass biopsy/resection was determined on the basis of the clinical documentation, and whether the radiologist's report favored a primary cord mass or an ISCM was ascertained.

For the comparison group, the pathologic data base at our institution was searched for patients with pathologically proved primary spinal cord intramedullary masses $(n=84)$ with 1 of the following histopathologies: ependymoma (consecutive from 2009 to 2011), astrocytoma (consecutive from 2006 to 2011), hemangioblastoma (consecutive from 2000 to 2011), ganglioglioma (consecutive from 2006 to 2011), and cavernous malformation (consecutive from 1998 to 2011). The varying date ranges were chosen so that the final size of the comparison group was approximately the same as the ISCM group. Patients lacking available pretreatment MR imaging were excluded.

\section{MR Imaging Review}

Two radiologists reviewed the MR imaging examinations of all patients in consensus at an electronic workstation: one (F.E.D.), a neuroradiology faculty member with an ABR certification and a Certificate of Added Qualification in neuroradiology and in fulltime neuroradiology practice; and the other (J.B.R.), a current neuroradiology fellow with an ABR certification. The examinations included MR imaging from predominantly our and several outside institutions. All available pulse sequences were reviewed,

with the typical examination including sagittal T1- and T2weighted, axial T2-weighted, and postgadolinium sagittal and axial T1-weighted images. The presence or absence of the following imaging features was analyzed by using pre- and postgadolinium T1-weighted images: 1) gadolinium enhancement, 2) rim sign, and 3) flame sign. The rim sign was defined as a complete or partial thin peripheral rim of gadolinium enhancement more intense than the central enhancement of a noncystic/necrotic lesion (Fig 1). When present, it was rated as either partial or complete/ near-complete. The flame sign was an ill-defined flame-shaped region of gadolinium enhancement at the superior and/or inferior margin of an otherwise well-defined lesion (Fig 2). Both the rim and flame signs can be present in the same lesion (Fig 3). For ISCMs, the maximum size of the enhancing lesion (including the flame sign, if present) was measured in millimeters, by using the superior-inferior dimension on sagittal T1-weighted postgadolinium scans in all cases.

\section{Histopathologic Review}

The histopathologic slides of the 5 patients in whom the ISCM had been biopsied/resected were reviewed by a board-certified neuropathologist (C.G.). The neuropathologist was asked to assess the margins of the tumor for any possible correlates of the rim and flame signs. 

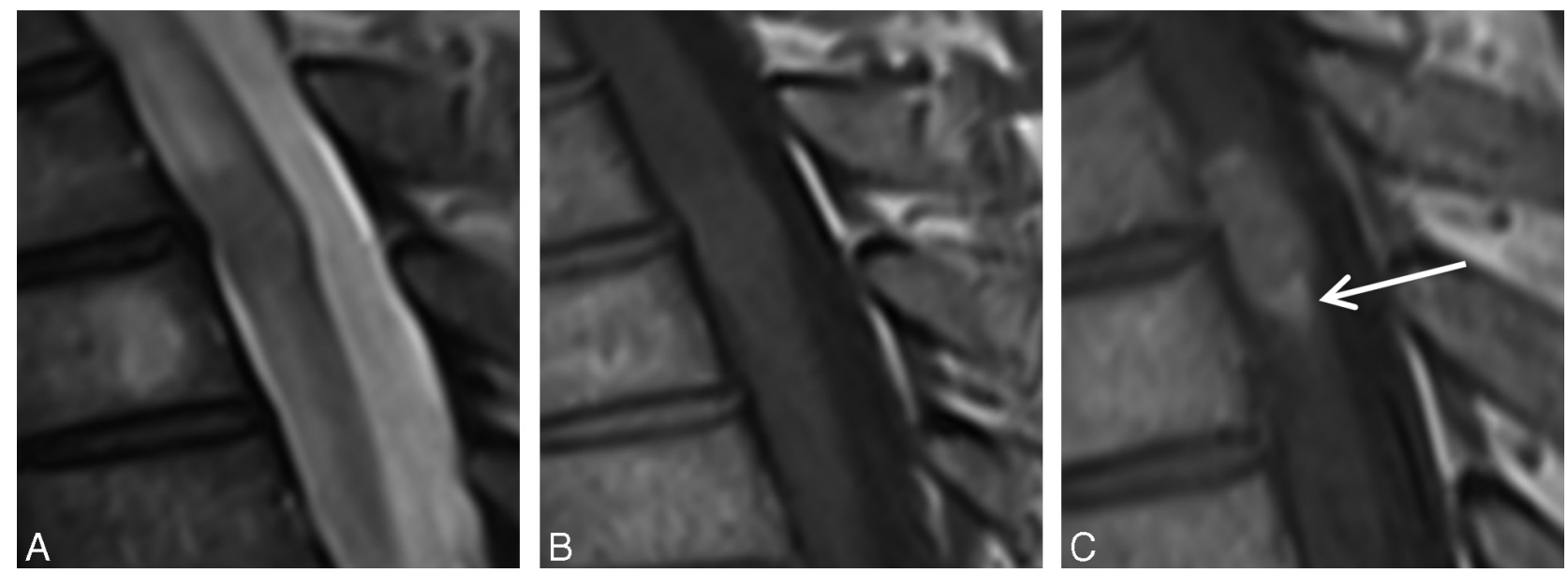

FIG 2. Flame sign. A 55-year-old man with metastatic small cell lung carcinoma who presented with severe midback pain, progressive weakness, and altered sensation in both lower extremities. MR imaging of the thoracic spine demonstrates intramedullary spinal cord metastasis at T3-4. Sagittal T2-weighted (A), T1-weighted (B), and postcontrast T1-weighted (C) images. Note the flame sign (arrow, C): an ill-defined flame-shaped region of enhancement at the inferior margin of the otherwise well-defined mass.
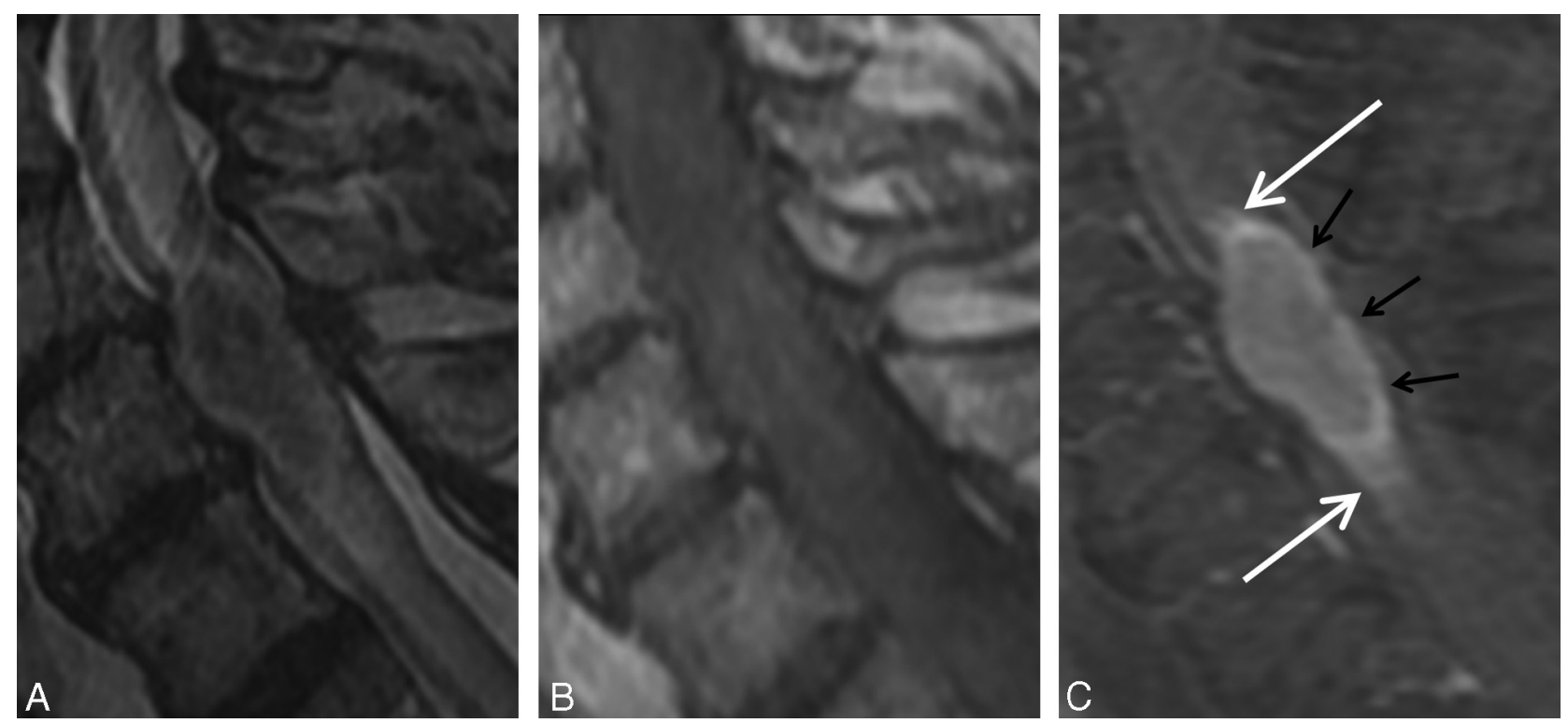

FIG 3. Concurrent rim and flame signs. A 68-year-old man with metastatic non-small cell lung carcinoma who presented with progressive bilateral lower extremity weakness. MR imaging of the cervical spine demonstrates an intramedullary spinal cord metastasis at C6. Sagittal T2-weighted $(A)$, T1-weighted $(B)$, and postcontrast T1-weighted fat-suppressed $(C)$ images. Note the enhancing intramedullary mass with a thin rim of more intense enhancement (rim sign; black arrows, $C$ ) as well as the ill-defined flame-shaped regions of enhancement at the superior and inferior margins of the mass (flame sign; white arrows, $C$ ).

\section{Clinical Review}

The electronic medical records were reviewed to identify those patients with an ISCM in whom: the cord lesion was identified before a diagnosis of systemic metastatic disease, radiation therapy for their primary malignancy had included the region of spinal cord in which the ISCM developed, and/or systemic steroid therapy was ongoing at the time of MR imaging.

\section{Statistical Analysis}

The frequency of rim and/or flame signs in ISCMs and primary cord masses was compared by using the $\chi^{2}$ test. We calculated the sensitivity of the rim and/or flame signs for diagnosing ISCM, assuming ISCMs with these signs to be true-positives and those without, false-negatives. We calculated the specificity of these signs for diagnosing ISCM, assuming primary cord masses with these findings to be false-positives and those without, truenegatives. For the ISCMs, a $t$ test, assuming unequal variances, was used to analyze the correlation between the presence of rim and/or flame signs and mean enhancing lesion maximum size; and the $\chi^{2}$ test was used to analyze the correlation between the presence of rim and/or flame signs and either radiation therapy for primary malignancy or systemic steroid therapy. Statistical significance was set at a $P$ value of $<.05$, and analysis was conducted by using JMP Software, Version 7.0 (SAS Institute, Cary, North Carolina).

\section{RESULTS}

\section{Histopathologic Characteristics of ISCM}

Nine (16\%) of the 58 patients with potential ISCMs were excluded due to lack of available gadolinium-enhanced pretreatment digi- 
Table 1: Enhancement characteristics of intramedullary spinal cord metastases and primary masses (per-lesion basis)

\begin{tabular}{|c|c|c|c|c|c|c|c|}
\hline & $\begin{array}{l}\text { Intramedullary } \\
\text { Spinal Cord } \\
\text { Metastases } \\
(n=64)\end{array}$ & $\begin{array}{c}\text { Primary } \\
\text { Spinal Cord } \\
\text { Masses } \\
(n=64)\end{array}$ & $\begin{array}{l}\text { Ependymoma } \\
\qquad(n=21)\end{array}$ & $\begin{array}{c}\text { Astrocytoma } \\
(n=21)\end{array}$ & $\begin{array}{l}\text { Hemangioblastoma } \\
\qquad(n=11)\end{array}$ & $\begin{array}{l}\text { Ganglioglioma } \\
\qquad(n=5)\end{array}$ & $\begin{array}{l}\text { Cavernous } \\
\text { Malformation } \\
\quad(n=6)\end{array}$ \\
\hline No enhancement & $1(2 \%)$ & $10(16 \%)$ & 0 & $6(29 \%)$ & 0 & $1(20 \%)$ & $3(50 \%)$ \\
\hline Rim sign & $21(33 \%)$ & $2(3 \%)$ & 0 & 0 & $2(18 \%)$ & 0 & 0 \\
\hline Flame sign & $19(30 \%)$ & $2(3 \%)$ & $1(5 \%)$ & $1(5 \%)$ & 0 & 0 & 0 \\
\hline Rim without flame sign & $9(14 \%)$ & $2(3 \%)$ & 0 & 0 & $2(18 \%)$ & 0 & 0 \\
\hline Flame without rim sign & $7(11 \%)$ & $2(3 \%)$ & $1(5 \%)$ & $1(5 \%)$ & 0 & 0 & 0 \\
\hline Only rim or flame sign ${ }^{a}$ & $16(25 \%)$ & $4(6 \%)$ & $1(5 \%)$ & $1(5 \%)$ & $2(18 \%)$ & 0 & 0 \\
\hline Both rim and flame signs & $12(19 \%)$ & 0 & 0 & 0 & 0 & 0 & 0 \\
\hline At least rim or flame sign ${ }^{b}$ & $28(44 \%)$ & $4(6 \%)$ & $1(5 \%)$ & $1(5 \%)$ & $2(18 \%)$ & 0 & 0 \\
\hline
\end{tabular}

a Sum of the 2 preceding rows (lesions that demonstrated only 1 of the 2 signs).

${ }^{\mathrm{b}}$ Sum of the 2 preceding rows (lesions that demonstrated at least 1 of the 2 signs, whether alone or in combination with the other sign)

Table 2: Rim and flame signs in intramedullary spinal cord metastases (per-patient basis)

\begin{tabular}{|c|c|c|c|c|c|}
\hline & Rim Sign Only & Flame Sign Only & Both Signs & Neither Sign & At Least One Sign \\
\hline All ISCMs $(n=45)$ & $9(20 \%)$ & $6(13 \%)$ & $12(27 \%)$ & $18(40 \%)$ & $27(60 \%)$ \\
\hline Lung carcinoma $(n=22)$ & $6(27 \%)$ & $3(14 \%)$ & $8(36 \%)$ & $5(23 \%)$ & $17(77 \%)$ \\
\hline Breast carcinoma $(n=6)$ & 0 & 0 & $1(17 \%)$ & $5(83 \%)$ & $1(17 \%)$ \\
\hline Melanoma $(n=5)$ & 0 & $1(20 \%)$ & $1(20 \%)$ & $3(60 \%)$ & $2(40 \%)$ \\
\hline CNS origin $(n=4)$ & 0 & 0 & 0 & $4(100 \%)$ & 0 \\
\hline Renal cell carcinoma $(n=2)$ & 0 & $1(50 \%)$ & $1(50 \%)$ & 0 & $2(100 \%)$ \\
\hline Other $(n=6)$ & $3(50 \%)$ & $1(17 \%)$ & $1(17 \%)$ & $1(17 \%)$ & $5(83 \%)$ \\
\hline $\begin{array}{l}\text { ISCM identified prior to systemic metastatic } \\
\text { disease diagnosis }(n=10)\end{array}$ & $3(30 \%)$ & $2(20 \%)$ & $4(40 \%)$ & $1(10 \%)$ & $9(90 \%)$ \\
\hline $\begin{array}{l}\text { Radiation therapy for primary malignancy } \\
\text { included relevant spinal cord }(n=5)\end{array}$ & 0 & $1(20 \%)$ & $3(60 \%)$ & $1(20 \%)$ & $4(80 \%)$ \\
\hline $\begin{array}{l}\text { Ongoing systemic steroid therapy at time } \\
\text { of MRI ( } n=12)\end{array}$ & $2(17 \%)$ & $2(17 \%)$ & $3(25 \%)$ & $5(42 \%)$ & $7(58 \%)$ \\
\hline
\end{tabular}

tal MR imaging examinations. Four (8\%) of the remaining 49 patients were excluded because alternative diagnoses were being considered clinically and ISCM was never formally diagnosed. This process yielded a final ISCM study population of 45 (92\%) of the remaining 49 patients, with 64 lesions. In 5 (11\%) of these 45 patients with ISCM, the diagnosis of a solitary ISCM was proved via cord mass biopsy/resection. In an additional 39 (87\%) of the 45 patients with ISCM, pathologic proof of the systemic malignancy had been obtained from the primary site or a metastatic site outside the spinal cord. One (2\%) of the 45 patients died before any sampling but was clinically presumed to have an ISCM from lung carcinoma, given a classic radiographic pattern of dominant primary lung mass, with multiple pulmonary metastases, hilar and mediastinal adenopathy, distant metastases, a 50 pack-year smoking history, and a family history of lung cancer.

Thirty-six $(80 \%)$ of 45 patients had a solitary ISCM, while 9 (20\%) of 45 demonstrated $>1$ ISCM. The primary malignancies in the 45 patients with 64 lesions were the following: lung carcinomas in $22(49 \%)$ patients, 36 (56\%) lesions; breast carcinomas in $6(12 \%)$ patients, $11(17 \%)$ lesions; melanomas in $5(11 \%)$ patients, $5(8 \%)$ lesions; CNS-origin primaries in $4(9 \%)$ patients, $4(6 \%)$ lesions; renal cell carcinomas in $2(4 \%)$ patients, $2(3 \%)$ lesions; and 1 each from several other histologies (anaplastic thyroid carcinoma, salivary ductal carcinoma, neuroendocrine carcinoma, ovarian adenocarcinoma, lymphoma, and prostate carcinoma) for a total of $6(13 \%)$ patients, $6(9 \%)$ lesions. Of the 4 patients with CNS-origin primaries, 2 patients had both intramedullary and leptomeningeal spinal metastases, 1 from a pos- terior fossa medulloblastoma and 1 from an esthesioneuroblastoma; and 2 patients had ISCM only, 1 from a posterior fossa medulloblastoma and 1 from a cerebral glioblastoma.

\section{Histopathologic Characteristics of Primary Spinal Cord Masses}

For the comparison group of patients with pathologically proved primary spinal cord intramedullary masses, 20 (24\%) of 84 patients were excluded for lack of available pretreatment MR imaging. The final primary spinal cord mass group consisted of the remaining $64(76 \%)$ of 84 patients, distributed as follows: ependymoma in 21 (33\%) (20 WHO grade II and 1 grade I [myxopapillary] that involved the conus), astrocytoma in 21 (33\%) (10 pilocytic astrocytomas, 11 infiltrating astrocytomas [5 grade II, 5 grade III, and 1 grade IV]), hemangioblastoma in 11 (17\%), ganglioglioma in 5 (8\%) (4 grade I; 1 anaplastic ganglioglioma [anaplastic astrocytoma, grade III, arising in a background of low-grade ganglioglioma]), and cavernous malformation in $6(9 \%)$.

\section{Rim and Flame Signs in ISCM}

Sixty-three (98\%) of the 64 ISCMs enhanced with gadolinium. Table 1 demonstrates the per-lesion prevalence of the rim and flame signs in ISCMs compared with primary cord masses. On a per-patient basis (Table 2), the rim sign was present in 21 (47\%) of 45 patients with ISCMs and in isolation without the flame sign in $9(20 \%)$ of 45 patients (Fig 1). The flame sign was present in 18 $(40 \%)$ of 45 patients and in isolation without the rim sign in 6 

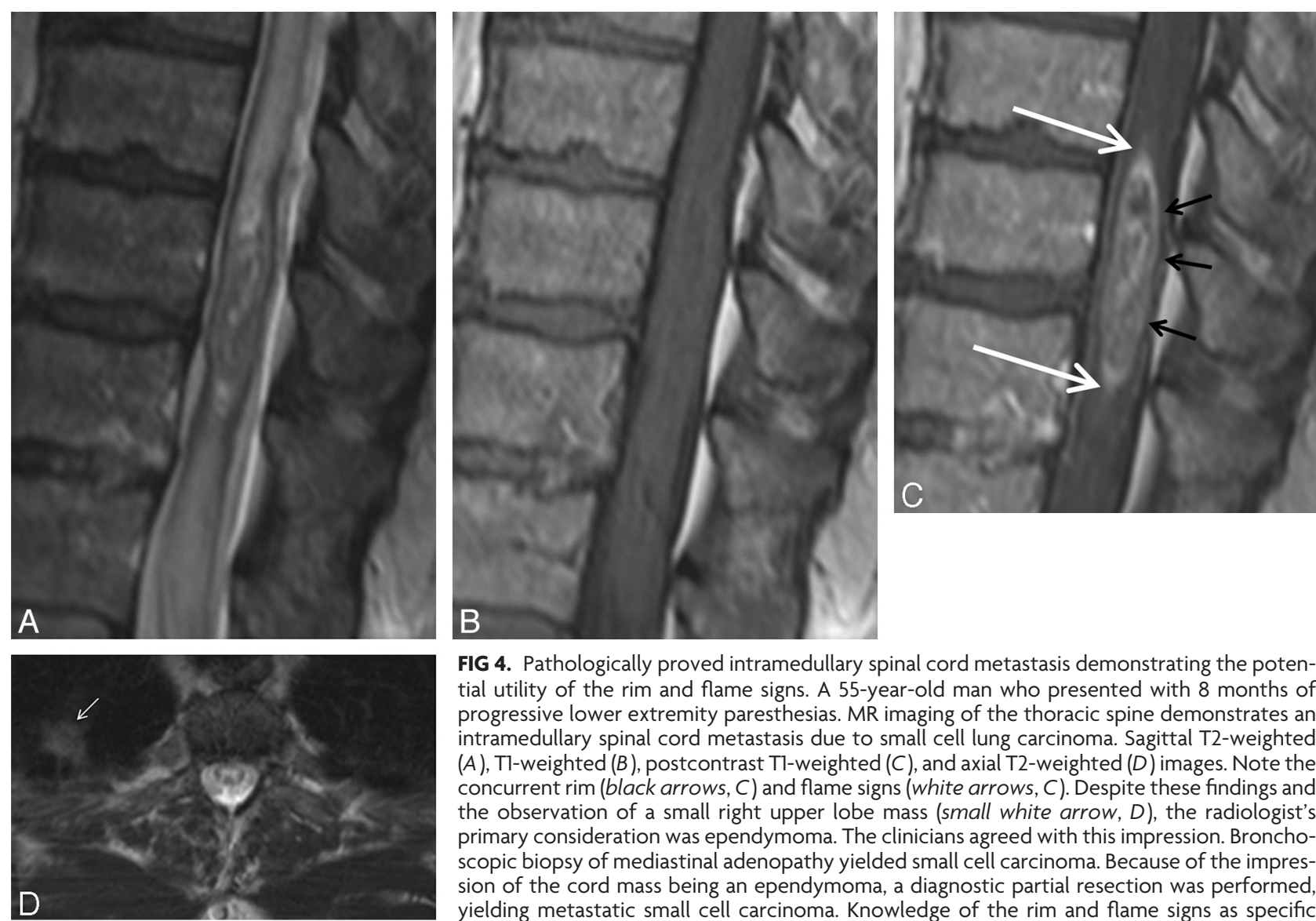

FIG 4. Pathologically proved intramedullary spinal cord metastasis demonstrating the potential utility of the rim and flame signs. A 55-year-old man who presented with 8 months of progressive lower extremity paresthesias. MR imaging of the thoracic spine demonstrates an intramedullary spinal cord metastasis due to small cell lung carcinoma. Sagittal T2-weighted $(A)$, T1-weighted (B), postcontrast T1-weighted (C), and axial T2-weighted (D) images. Note the concurrent rim (black arrows, $C$ ) and flame signs (white arrows, $C$ ). Despite these findings and the observation of a small right upper lobe mass (small white arrow, $D$ ), the radiologist's primary consideration was ependymoma. The clinicians agreed with this impression. Bronchoscopic biopsy of mediastinal adenopathy yielded small cell carcinoma. Because of the impression of the cord mass being an ependymoma, a diagnostic partial resection was performed, yielding metastatic small cell carcinoma. Knowledge of the rim and flame signs as specific findings of ISCM could potentially have avoided diagnostic biopsy.

(13\%) of 45 patients (Fig 2). The rim and flame signs were present concurrently (Fig 3) in $12(27 \%)$ of 45 patients, and at least 1 of the 2 signs was present in 27 (60\%) of 45 patients. Eighteen (40\%) of 45 patients did not exhibit either imaging sign, including the 1 patient with the completely nonenhancing ISCM. When present, the rim sign was complete/near-complete in $13(62 \%)$ of 21 and partial in $8(38 \%)$ of 21 lesions. When present, the flame sign was seen superiorly only in 1 (5\%) of 19 lesions, inferiorly only in 4 $(21 \%)$ of 19 , and at both ends in $14(74 \%)$ of 19 . None of the 4 CNS-origin ISCMs demonstrated either the rim or flame sign (Table 2).

Regarding the 5 (11\%) of 45 solitary ISCMs that were directly pathologically proved, 2 (40\%) of 5 demonstrated the rim and flame signs; 2 (40\%) of 5, the rim sign only; and 1 (20\%), the flame sign only. Thus, all 5 (100\%) of 5 of these lesions demonstrated at least either the rim or the flame sign. In 1 (20\%) of these 5 lesions, the surgical resection was performed primarily for therapeutic effect. In 4 (80\%) of these 5 lesions, it was performed primarily for diagnostic purposes: The rim sign was present in 2 cases, and both the rim and flame signs, in the other 2 (Fig 4). In 3 (60\%) of the 5 lesions, the radiologist's MR imaging report favored primary cord neoplasm over ISCM (Fig 4): One demonstrated a rim sign; 1, a flame sign; and 1, both signs. No histopathologic correlates to the rim or flame signs were identified in these 5 patients, including no evidence for a tumor capsule. This analysis was limited because all 5 tumors appeared highly fragmented. Thus, the tumor margins and relationship to the surrounding cord parenchyma could not be assessed.
In $10(22 \%)$ of the 45 patients with ISCMs, the cord lesion was identified before a diagnosis of systemic metastatic disease. Five (11\%) of the 45 patients with ISCMs had undergone radiation therapy for their primary malignancy that included the region of the spinal cord in which the ISCM developed. Twelve (27\%) of the 45 patients with ISCMs were on systemic steroid therapy at the time of MR imaging. The frequencies of the signs in these clinical subgroups are displayed in Table 2. Neither radiation therapy for the primary malignancy involving the relevant region of spinal cord nor steroid therapy at the time of MR imaging statistically correlated with the frequency of the rim and/or flame signs $(P$ values all $\geq .05$ ).

\section{Rim and Flame Signs in Primary Cord Masses}

Fifty-four (84\%) of 64 primary cord masses enhanced with gadolinium. Table 1 demonstrates the per-lesion prevalence of the rim and flame signs in primary cord masses compared with ISCMs. In the 2 primary cord lesions with the rim sign, both hemangioblastomas, the rim sign was partial (2 of 2; 100\%) (Fig $5 A,-B)$. In both of the primary cord lesions with the flame sign, 1 an ependymoma and 1 an astrocytoma, the flame sign was present at only 1 margin of the lesions ( 2 of $2 ; 100 \%$ ) (Fig $5 C,-D$ ).

\section{Diagnostic Utility of Rim and Flame Signs}

The rim and flame signs, alone and in combination, were seen more frequently in ISCMs than in primary cord masses $(P<$ .0001 for each). The sensitivities of the rim and flame signs on a per lesion basis for the diagnosis of ISCM were the following: rim 

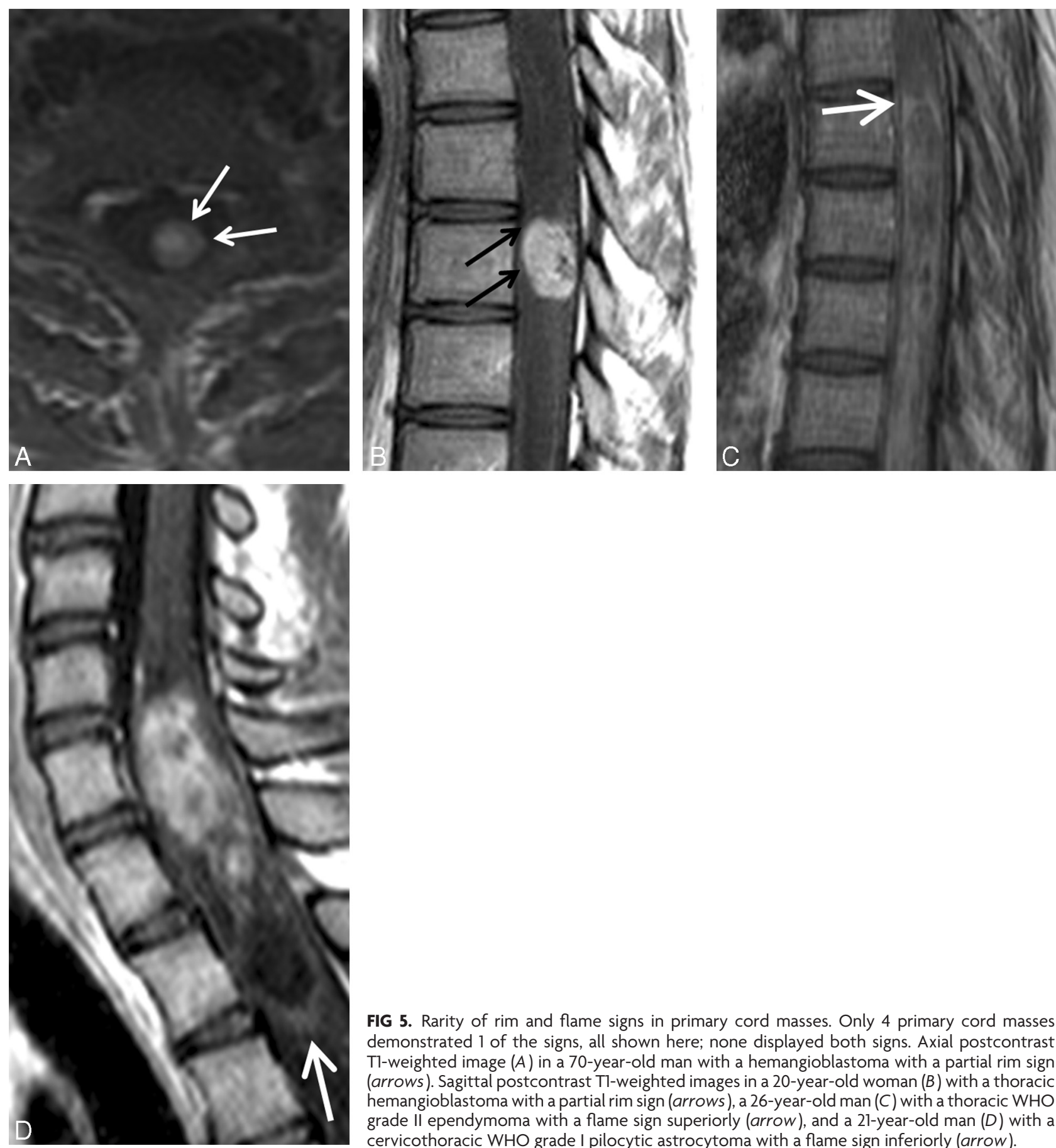

FIG 5. Rarity of rim and flame signs in primary cord masses. Only 4 primary cord masses demonstrated 1 of the signs, all shown here; none displayed both signs. Axial postcontrast T1-weighted image $(A)$ in a 70-year-old man with a hemangioblastoma with a partial rim sign (arrows). Sagittal postcontrast T1-weighted images in a 20-year-old woman (B) with a thoracic hemangioblastoma with a partial rim sign (arrows), a 26-year-old man (C) with a thoracic WHO grade II ependymoma with a flame sign superiorly (arrow), and a 21-year-old man (D) with a cervicothoracic WHO grade I pilocytic astrocytoma with a flame sign inferiorly (arrow).

sign, 33\% (21/64); flame sign, 19\% (19/64); at least 1 sign, $44 \%$ (28/64); and both signs concurrently, 19\% (12/64). The sensitivities of the rim and flame signs on a per-patient basis were the following: rim sign, $47 \%$ (21/45); flame sign, 40\% (18/45); at least 1 sign, $60 \%$ (27/45); and both signs concurrently, 27\% (12/45). The specificities of the rim and flame signs on a per-lesion (and per-patient) basis for the diagnosis of ISCM were the following: rim sign, 97\% (62/64); flame sign, 97\% (62/64); at least 1 sign, 94\% (60/64); and both rim and flame signs concurrently, 100\% (64/64).

\section{Maximum ISCM Size and Correlation with the Presence of the Rim or Flame Sign}

Among the ISCM group, the rim sign and the flame sign each individually positively correlated with the mean measured enhancing lesion size (superior-inferior direction), with statistical significance $(P=.0065$ and $P=.0012$, respectively). The mean enhancing lesion sizes for ISCMs with-versus-without these signs, respectively, were 31 versus $14 \mathrm{~mm}$ for the rim sign and 34 versus $14 \mathrm{~mm}$ for the flame sign.

AJNR Am J Neuroradiol 34:908-915 Apr 2013 www.ajnr.org 


\section{DISCUSSION}

We describe 2 peripheral enhancement features on MR imaging specific for non-CNS-origin ISCMs compared with primary cord masses: a more intense thin rim of peripheral enhancement around an enhancing lesion and an ill-defined flame-shaped region of enhancement at the superior/inferior margins. These rim and flame signs were prevalent in ISCMs and rare in primary cord masses. Among spinal cord masses, the rim and flame signs thus have high specificity for spinal cord metastases. These signs were not seen in any of the 4 ISCMs of CNS origin.

These results have clinical relevance for several reasons. We expect that knowledge of these specific findings will improve the radiologist's confidence in diagnosing ISCM. This could enable earlier clinical diagnosis and appropriate treatment, which, according to some literature studies, can improve survival in patients with ISCM. ${ }^{5,6}$ In our study, at least 1 sign was present in almost all (90\%) patients with ISCMs, who at the time of MR imaging had not yet been diagnosed with systemic metastatic malignancy. In addition, recognition of the rim and/or flame signs may help avoid diagnostic resection, which is performed in some ISCM cases. Supporting this notion is the fact that of our 5 patients who underwent ISCM resection, resection was performed not for therapeutic purposes but primarily because of diagnostic uncertainty. In 3 of these 5 patients, the radiologist's MR imaging report favored primary cord neoplasm over ISCM, despite the presence of at least 1 of the 2 signs in each case.

Our study adds to the existing literature on ISCMs. To our knowledge, this is the largest series of ISCMs analyzing postgadolinium MR imaging findings of these lesions. The largest currently available series consists of 40 patients (only 25 imaged with gadolinium-enhanced MR imaging). ${ }^{7}$ The next largest series include studies with 19 patients, ${ }^{6} 12$ patients, ${ }^{8} 12$ patients (only 6 imaged with unenhanced MR imaging), ${ }^{9}$ and 7 patients. ${ }^{10}$ Most interesting, in 1 of these prior studies ${ }^{10}$ a "ring-enhancing" ISCM (their Fig 2) actually demonstrated apparent flame and possible rim signs. In fact, on the basis of our review of the relevant literature, other examples of unreported apparent flame and rim signs are evident. ${ }^{6-8,11-15}$ We believe that this finding lends further validity to our findings that the rim and flame signs are common in ISCMs.

A limitation of this study is that the MR images were interpreted by a neuroradiology staff member and fellow in consensus. However, in doing so, it was our goal to simulate clinical academic neuroradiology practice and report on the initial observation of new imaging findings; these 2 scenarios have been proposed to be suitable situations in which consensus review can be justified. ${ }^{16}$ The limitations of our study also include its retrospective nature, the heterogeneity in the MR imaging technique given that the MR images were from several different decades and from different institutions, the fact that the reviewers were not blinded to histopathology, and not all potential cord mass pathologies being considered. However, prospective studies of the rare entity of ISCM with a standardized MR imaging technique would prove difficult. The readers of our study carefully scrutinized all cases for these findings, regardless of lesion type. We believe that our comparison group of pathologically proved primary cord masses was representative of the lesions that could most commonly be confused on MR imaging for ISCMs. Our primary cord lesions consisted mostly (42 [66\%] of 64) of the 2 most common types of primary cord tumors, ependymomas and astrocytomas. It also included several nonenhancing primary cord tumors, which are known to occur, particularly among astrocytomas. ${ }^{17}$

Future work could include surgical and additional radiologicpathologic correlation of both the rim and flame signs. Histopathologic analysis in ISCM cases with less fragmented tumor samples might be more revealing with regard to microscopic findings at the tumor margins. Because there are various degrees of resectability encountered by neurosurgeons attempting to remove ISCMs, ${ }^{6}$ it would also be interesting to analyze whether the rim sign predicts the presence of a cleavage plane and, in turn, an easier, more complete resection. The degree to which varied pathologic processes, such as ischemia, demyelination, and tumor infiltration, contribute to the flame sign would also be of interest and could be studied specifically at postmortem in future cases. Indeed, the pathologic findings in 3 of 5 ISCM cases in 1 series included "central ischemic infarction...cephalad and caudad to the tumor metastasis." 18

\section{CONCLUSIONS}

We describe 2 peripheral enhancement features on MR imaging specific for non-CNS-origin ISCM compared with primary cord masses: a more intense thin rim of peripheral enhancement around an enhancing lesion (rim sign) and an ill-defined flameshaped region of enhancement at the superior/inferior margins (flame sign). The prevalences of these signs in ISCMs and primary cord masses in this series, respectively, were the following: rim sign, 33\% (21 of 64) and 3\% (2 of 64) $(P<.0001)$; and flame sign, $30 \%$ (19 of 64$)$ and $3 \%(2$ of 64$)$, respectively $(P<.0001)$. The prevalences were even higher in cases of ISCMs in which the cord lesion was identified before a diagnosis of systemic metastatic disease: $70 \%$ ( 7 of 10) with a rim sign, $60 \%$ (6 of 10) with a flame sign, and $90 \%$ ( 9 of 10) with at least 1 of the signs. Knowledge of these findings should increase radiologists' confidence in diagnosing ISCMs, thus enabling earlier diagnosis and treatment. Diagnostic biopsy/resection, which is still performed even in the MR imaging era for some ISCMs, may be obviated in some cases.

\section{REFERENCES}

1. Costigan DA, Winkelman MD. Intramedullary spinal cord metastasis: a clinicopathological study of 13 cases. J Neurosurg 1985; 62:227-33

2. Chason JL, Walker FB, Landers JW. Metastatic carcinoma in the central nervous system and dorsal root ganglia: a prospective autopsy study. Cancer 1963;16:781-87

3. Mut M, Schiff D, Shaffrey ME. Metastasis to nervous system: spinal epidural and intramedullary metastases. J Neurooncol 2005;75: $43-56$

4. Connolly ES Jr, Winfree CJ, McCormick PC, et al. Intramedullary spinal cord metastasis: report of three cases and review of the literature. Surg Neurol 1996;46:329-37, discussion 337-38

5. Kalayci M, Cagavi F, Gul S, et al. Intramedullary spinal cord metastases: diagnosis and treatment-an illustrated review. Acta Neurochir (Wien) 2004;146:1347-54, discussion 1354

6. Dam-Hieu P, Seizeur R, Mineo JF, et al. Retrospective study of 19 patients with intramedullary spinal cord metastasis. Clin Neurol Neurosurg 2009;111:10-17 
7. Schiff D, O'Neill BP. Intramedullary spinal cord metastases: clinical features and treatment outcome. Neurology 1996;47:906-12

8. Lee SS, Kim MK, Sym SJ, et al. Intramedullary spinal cord metastases: a single-institution experience. J Neurooncol 2007;84: 85-89

9. Post MJ, Quencer RM, Green BA, et al. Intramedullary spinal cord metastases, mainly of nonneurogenic origin. AJR Am J Roentgenol 1987;148:1015-22

10. Watanabe M, Nomura T, Toh E, et al. Intramedullary spinal cord metastasis: a clinical and imaging study of seven patients. J Spinal Disord Tech 2006;19:43-47

11. Crowley RW, Sherman JH, Le BH, et al. Intramedullary spinal cord metastasis from bladder carcinoma: case report. Neurosurgery. 2008;63:E611-12, discussion E612

12. Fakih M, Schiff D, Erlich R, et al. Intramedullary spinal cord metastasis (ISCM) in renal cell carcinoma: a series of six cases. Ann Oncol 2001;12:1173-77
13. Ogino M, Ueda R, Nakatsukasa M, et al. Successful removal of solitary intramedullary spinal cord metastasis from colon cancer. Clin Neurol Neurosurg 2002;104:152-56

14. Grasso G, Meli F, Patti R, et al. Intramedullary spinal cord tumor presenting as the initial manifestation of metastatic colon cancer: case report and review of the literature. Spinal Cord 2007; 45:793-96

15. Sze G, Krol G, Zimmerman RD, et al. Intramedullary disease of the spine: diagnosis using gadolinium-DTPA-enhanced MR imaging. AJR Am J Roentgenol 1988;151:1193-204

16. Bankier AA, Levine D, Halpern EF, et al. Consensus interpretation in imaging research: is there a better way? Radiology 2010;257:14-17

17. White JB, Miller GM, Layton KF, et al. Nonenhancing tumors of the spinal cord. J Neurosurg Spine 2007;7:403-07

18. Hashizume Y, Hirano A. Intramedullary spinal cord metastasis. Pathologic findings in five autopsy cases. Acta Neuropathol 1983;61: $214-18$ 